\title{
Clostridium difficile Infection Leading to Intestinal Pneumatosis in a Patient with a Recent Diagnosis of Pancreatic Cancer Local Recurrence: A Case Report and Literature Review
}

\author{
Angel Ayumi Tome Uchiyama ${ }^{a}$ Moisés de Souza Martins Lopes ${ }^{b}$ \\ Maira Nacimbem Marzinotto Vana ${ }^{c}$ Renata D'Alpino Peixoto ${ }^{d}$ \\ ${ }^{a}$ Oncology, Hospital Alemão Oswaldo Cruz, São Paulo, Brazil; boncology, Hospital Alemão \\ Oswaldo Cruz, São Paulo, Brazil; ' 'Gastroenterology, Hospital Alemão Oswaldo Cruz, São \\ Paulo, Brazil; 'Oncology, Centro Paulista de Oncologia - Grupo Oncoclínicas, São Paulo, \\ Brazil
}

\section{Keywords}

Clostridium difficile $\cdot$ Intestinal pneumatosis, $\cdot$ Pancreatic cancer

\begin{abstract}
Clostridium difficile infection (CDI) causing pneumatosis intestinalis $(\mathrm{PI})$ is a rare event, described mostly in immunocompromised patients. We present the case of a 65 -year-old female diagnosed with adenocarcinoma of the pancreas who underwent a duodenopancreatectomy with lymphadenectomy and adjuvant gemcitabine and capecitabine. Four months after the end of chemotherapy, she experienced abdominal pain and intermittent diarrhea which became aggravated within 6 months. CT scans revealed diffuse intestinal pneumatosis and recurrence of ductal adenocarcinoma. We hypothesize that local pancreatic cancer recurrence may lead to gastrointestinal dysmotility with consequent increased risk for CDI. The patient had almost complete resolution of PI during the CDI treatment, thus we believe that the CDI was directly responsible for $\mathrm{PI}$ in this case.
\end{abstract}

\section{Introduction}

Pneumatosis intestinalis (PI) is defined by the finding of gas within the submucosa and/ or the subserosa of the large or small intestines on abdominal imaging, is a rare disorder. It may be associated with multiple causes, such as acquired immunodeficiency, transplant status, cancer treatment, scleroderma, cystic fibrosis, systemic lupus, inflammatory bowel 
disease, colitis, intestinal ischemia, vascular occlusion, trauma, bowel preparation, or just an asymptomatic radiographic finding [1]. Infectious agents are the major etiologic factor for PI development in immunocompromised hosts, such as cancer patients [2].

The prevalence of PI has been reported in $0.37 \%$ of patients undergoing abdominal CT, and it typically presents in the fifth to eighth decade of life [3, 4]. Although its clinical course is generally quite benign, with around $50 \%$ of cases spontaneously resolving, particularly when idiopathic, there is still a $20-40 \%$ mortality rate, emphasizing the need to differentiate between bowel ischemia and benign PI [3]. A previous large study including 500 patients with PI during a 10 -year period reported that $60 \%$ of the cases were classified as a benign disease, while $40 \%$ of them had a pathologic etiology [1].

The pathogenesis of PI remains unknown and several theories have been proposed. The mechanical theory of mucosal disruption predicates that gas dissects into the wall of the bowel through breaches in the integrity of a luminal surface and moves along mesenteric blood vessels [4]. Likewise, the bacterial theory postulate that gas formed by bacteria gains access to the submucosa through a break in the mucosa $[2,5,6]$. Supporters of the biochemical theory explain that intestinal bacteria produce an excessive amount of hydrogen which leads to increased pressure, causing gas to become trapped within the submucosa $[7,8]$. Those who advocate the immunosuppressive theory hypothesize that lymphoid depletion causes shrinkage of Peyer's patches, resulting in loss of structural integrity, which in turn permits dissection of gas into the bowel wall $[4,9]$.

Since most cases of PI are benign and asymptomatic, the majority of patients with this affection would probably never have been diagnosed. However, if patients are symptomatic they typically present with diarrhea, abdominal pain, distension, flatulence, rigidity, absent bowel sounds, tenesmus, and even peritonitis. Its complications include portal venous gas, pneumoperitoneum, volvulus, intestinal obstruction, perforation, and intussusception [10, 11]. While some patients can be observed without any aggressive intervention, others demand surgery for resection of ischemic bowel due to pathologic PI. Therefore, it is important to determine the correct management option, whether to perform an emergency laparotomy for an acute intra-abdominal catastrophe or to manage the case conservatively for rather benign cases [1]. Health care providers are required to use all of the clinical information available, including medical history, physical examination, laboratory findings (particularly lactic acidosis and elevated amylase), and the specifics of the radiographic presentation [12]. Physical examination remains a mainstay of initial evaluation because patients with peritoneal signs typically mandate operative exploration to rule out intestinal perforation or ischemia. On the other hand, PI observed in imaging findings without leukocytosis, elevated lactic acid, and poor clinical examination findings could be treated conservatively. Management of this affection in non-life-threatening symptomatic patients includes antibiotics, elemental diet, and inhaled or hyperbaric oxygen. In asymptomatic patients, usually no therapy is indicated as intramural gas usually resolves spontaneously over time [11,13-15].

The incidence of PI among cancer patients and its main causes remains undetermined. Here we report the case of a patient with resected pancreatic adenocarcinoma who developed diarrhea and abdominal pain more than 1 year after completing adjuvant chemotherapy and was found to have diffuse PI on abdominal CT scan due to Clostridium difficile infection (CDI).

\section{Case Report/Case Presentation}

During a routine follow-up for gallbladder adenomyomatosis, a 65-year-old woman underwent an abdominal ultrasound which revealed a hypoechogenic lesion in the pancreatic head, measuring $2.2 \times 1.7 \mathrm{~cm}$. An abdominal MRI confirmed a solid hypovascularized mass in 
the uncinated pancreatic process, without lymphadenopathies or vascular involvement. CA19-9 was normal (12 UI/mL). In January 2018, she underwent a duodenopancreatectomy with lymphadenectomy. Pathology confirmed a pT3 pN1 (5/17 positive lymph nodes) ductal adenocarcinoma of the pancreas. Nine days after being discharged, the patient developed fever with rigors, followed by explosive diarrhea. She was admitted and tested positive for $C$. difficile, which was treated with orally administered metronidazole for 14 days with complete resolution of the infection.

From March to August 2018, she received adjuvant gemcitabine and capecitabine without any adverse events. Subsequently, she was asymptomatic and on routine follow-up. However, in December 2019, she started complaining of abdominal pain, especially in the left upper quadrant, associated with intermittent diarrhea and abdominal cramps. At that time, CT scans showed only discrete fat densification at the root of the mesenterium, which was stable according to the CT scans from 6 months before, and CA19-9 was normal. The abdominal pain and diarrhea aggravated and new CT scans were ordered in May 2020, revealing the emergence of diffuse intestinal pneumatosis along the colic frame as well as multiple small gaseous foci of pneumoperitoneum in the pericolic fat, as shown in Figures 1-3. In addition, the tissue located circumferentially to the emergence of the superior mesenteric artery became better defined, extending along its proximal segment, and at the level of the bifurcation of the celiac trunk, measuring up to $2.6 \mathrm{~cm}$. Given the suspicion of severe bowel infection, the patient was admitted and several tests were ordered, which confirmed CDI. Oral vancomycin was started with resolution of the diarrhea. Seven days later, there was almost complete resolution of the intestinal pneumatosis. In order to rule out locoregional recurrence, an echoendoscopy was requested, which unfortunately revealed ductal adenocarcinoma.

\section{Discussion}

C. difficile is an anaerobic, Gram-positive bacillus that colonizes the colon and transmits itself via the fecal-oral route [16]. CDI is the most common cause of health care-associated infection [17] and may lead to PI findings on imaging. There is a strong association between CDI and prior antibiotic use, especially with clindamycin, fluoroquinolones, and penicillins/ cephalosporins. Patients with CDI typically present with watery, non-bloody diarrhea, lowgrade fever, abdominal pain, and nausea. While CDI is becoming more common in all hospitalized patients, patients with cancer appear to be at an elevated risk. For instance, some

Fig. 1. CT scan of the abdomen revealing diffuse intestinal pneumatosis along the colic frame as well.

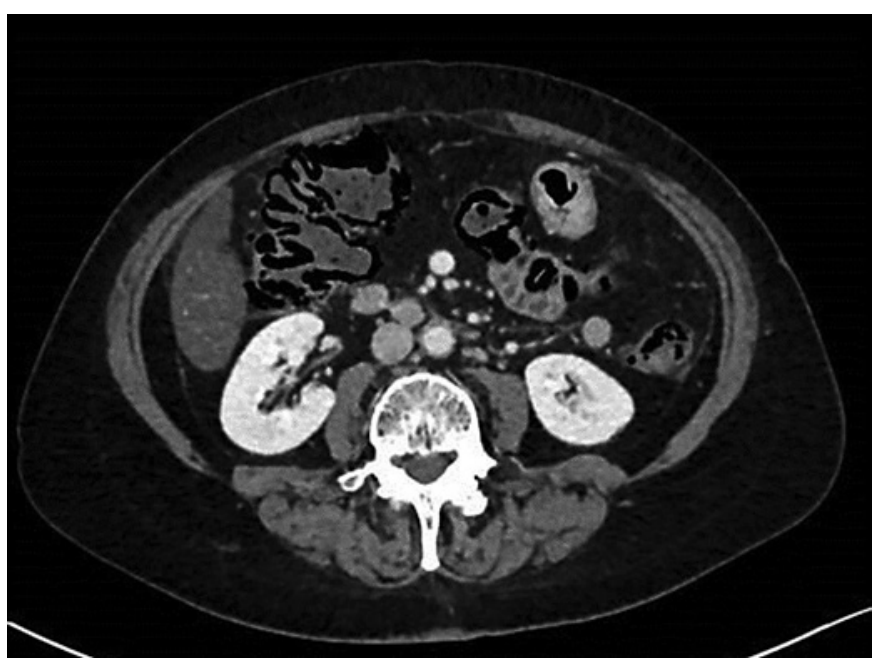


Fig. 2. CT scan of the abdomen revealing gas tracks along the bowel wall.

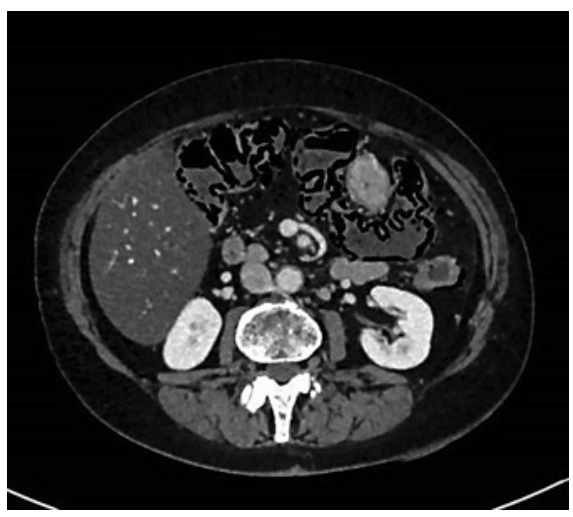

Fig. 3. CT scan of the abdomen revealing intestinal pneumatosis.

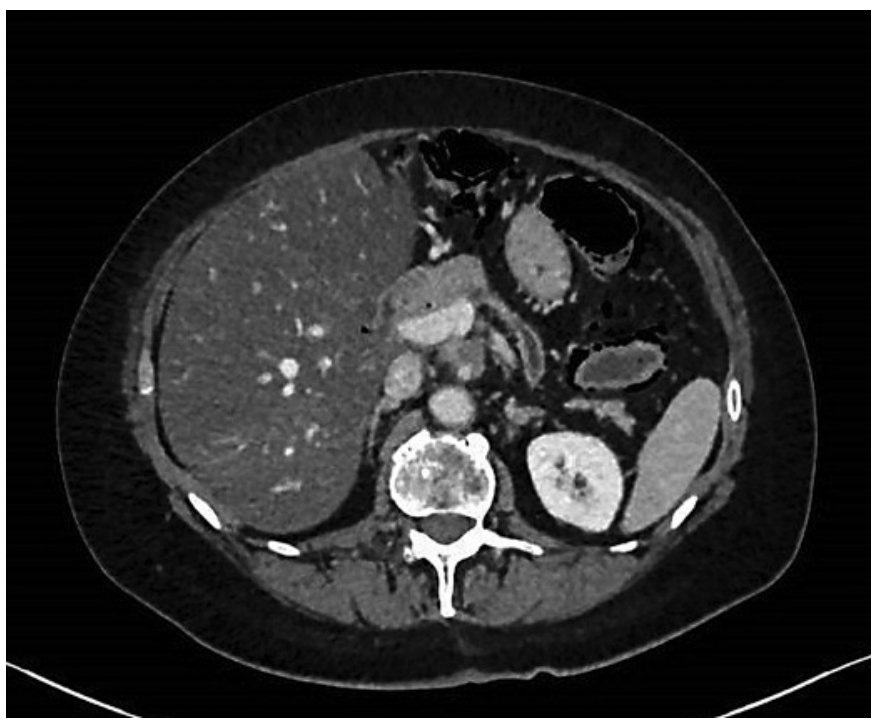

studies have reported that hospital-acquired $C$. difficile-associated diarrhea in patients with cancer was twice the rate reported for all patients in the USA (15.8 vs. 7.4 per 10,000 patients, respectively) [18]. Several factors may explain this risk, including increased exposure to antibiotics (for treatment or prophylaxis), advanced age (in some cases), immunocompromised state (due to cancer itself or chemotherapy), and frequent hospitalizations [19]. Severe CDI is usually accompanied by a white blood cell count of greater than $15,000 \mathrm{cell} / \mathrm{mL}$ or a serum creatinine greater than $1.5 \mathrm{mg} / \mathrm{dL}$; however, cancer patients may be neutropenic due to chemotherapy or the underlying malignancy, which might interfere with severity definition [20].

Chemotherapy often causes diarrhea as a consequence of treatment toxicity. This leads to gastrointestinal mucosal damage from decreased intestinal cell proliferation, increased apoptosis of crypt cells, and the immunosuppression state following chemotherapy predisposes to intestinal microbial imbalance [21]. Also, chemotherapy that disrupts the gut microbiome and causes mucositis may render germination of $C$. difficile spores more prevalent, leading to greater virulence. Studies have reported an association between the occurrence of CDI and specific chemotherapeutic agents such as 5-fluorouracil, DNA topoisomerase inhibitors, cisplatin, paclitaxel, and carboplatin $[18,22]$.

While CDI rates in patients with cancer vary between studies, they can be as high as $10 \%$ during a course of chemotherapy and up to a $20 \%$ risk overall [23]. One study found that 
patients with gastrointestinal tumors were at a decreased risk of CDI, while those with breast cancer had a higher risk [24]; however, it is unclear whether cancer subtypes confer a particularly high CDI risk. Furthermore, these patients have a variable presentation, with symptoms ranging from mild diarrhea to severe enterocolitis. They also tend to have worse outcomes, with higher mortality rates and prolonged hospital stays on average [25]. Compared with patients without cancer, the cure rates of CDI reported in the literature for oncology patients were significantly lower. Also, their median resolution times of diarrhea were longer, and recurrence rates higher. Evidence shows that cancer is an independent risk factor for recurrence of $C$. difficile infection [26, 27]. In the presented case, we hypothesized that local pancreatic cancer recurrence might have contributed some gastroparesis, which in turn led to CDI. At the time of the CDI with PI, the patient was not on chemotherapy for more than 1 year and had not received recent antibiotics.

Clinical practice guidelines for managing CDI were recently updated by the Infectious Disease Society of America (IDSA) [28]. Although no recommendations were specific to patients with cancer, many are applicable to this population. For hospitalized patients, contact precautions for at least $48 \mathrm{~h}$ after diarrhea resolves is recommended, but it is advised to be continued until medical discharge. Inpatients should also be placed on contact precautions as soon as CDI is suspected, and when the diagnosis is confirmed they should have their own bathroom. Treatment recommendations include oral vancomycin or fidaxomicin for 10 days instead of metronidazole for an initial episode of CDI. In the reported case, resolution of the symptoms and PI were obtained with oral vancomycin. A recent randomized control trial showed that fidaxomicin was non-inferior to oral vancomycin in the treatment of CDI and was associated with a lower rate of recurrence [20].

CDI causing PI is a rare phenomenon, described mostly in immunocompromised patients $[29,30]$. Although our patient had recurrence of the ductal adenocarcinoma, she was not under chemotherapy treatment at the time of the diagnosis. Since the patient had almost complete resolution of PI during the CDI treatment, we believe that the CDI was directly responsible for PI in this case.

\section{Conclusion}

The suspicion rate for CDI among cancer patients must be high due to its increased frequency, while imaging findings of PI may be a reflection of a CDI. We hypothesize that local pancreatic cancer recurrence may lead to gastrointestinal dysmotility with a consequent increased risk for CDI.

\section{Statement of Ethics}

Written informed consent was obtained from the patient for publication of this case report and any accompanying images.

\section{Conflict of Interest Statement}

The authors declare no conflicts of interest. 


\section{Case Reports in Oncology}

Case Rep Oncol 2021;14:1111-1117

DOI: $10.1159 / 000513003$

(c) 2021 The Author(s). Published by S. Karger AG, Basel www.karger.com/cro

\section{Funding Sources}

There are no funding sources to declare.

\section{Author Contributions}

A.A.T.U.: conception, design and data acquisition for the work. Main drafting of the case report and literature review. M.d.S.M.L.: design, acquisition and interpretation of data. Drafting of the case report. M.N.M.V.: analysis and interpretation of the data, and critical revision for important intellectual content. R.D.P.: conception of the case report, critical revision for important intellectual content, and final approval of the version to be published.

\section{References}

1 DuBose JJ, Lissauer M, Maung AA, Piper GL, O'Callaghan TA, Luo-Owen X, et al. Pneumatosis intestinalis predictive evaluation study (PIPES): a multicenter epidemiologic study of the Eastern Association for the Surgery of Trauma. J Trauma Acute Care Surg. 2013 Jul;75(1):15-23.

2 Schenk P, Madl C, Kramer L, Ratheiser K, Kranz A, Zauner C, et al. Pneumatosis intestinalis with Clostridium difficile colitis as a cause of acute abdomen after lung transplantation. Dig Dis Sci. 1998;43(11):2455.

3 Morris MS, Gee AC, Cho SD, Limbaugh K, Underwood S, Ham B, et al. Management and outcome of pneumatosis intestinalis. Am J Surg. 2008 May 1;195(5):679-3.

4 Pear BL. Pneumatosis intestinalis: a review. Radiology. 1998 Apr 1;207(1):13-9.

5 Yale CE, Balish E, Wu JP. The bacterial etiology of pneumatosis cystoides intestinalis. Arch Surg. 1974 Jul; 109(1):89-94.

6 Ellis BW. Symptomatic treatment of primary pneumatosis coli with metronidazole. Br Med J. 1980 Mar 15; 280(6216):763-4.

7 Hisamoto A, Mizushima T, Sato K, Haruta Y, Tanimoto Y, Tanimoto M, et al. Pneumatosis cystoides intestinalis after alpha-glucosidase inhibitor treatment in a patient with interstitial pneumonitis. Intern Med. 2006;45(2): 73-6.

8 Kojima K, Tsujimoto T, Fujii H, Morimoto T, Yoshioka S, Kato S, et al. Pneumatosis cystoides intestinalis induced by the $\alpha$-glucosidase inhibitor miglitol. Intern Med. 2010;49(15):1545-8.

9 Wood BJ, Kumar PN, Cooper C, Silverman PM, Zeman RK. Pneumatosis intestinalis in adults with AIDS: clinical significance and imaging findings. AJR Am J Roentgenol. 1995 Dec;165(6):1387-90.

10 Bailey J, Shaffer EA. Pneumatosis coli associated with pseudomembranous colitis in a patient following colonic surgery. Case Rep Med. 2010;2010:138369.

11 Khalil PN, Huber-Wagner S, Ladurner R, Kleespies A, Siebeck M, Mutschler W, et al. Natural history, clinical pattern, and surgical considerations of pneumatosis intestinalis. Eur J Med Res. 2009 Jun 18;14(6):231-9.

12 Knechtle SJ, Davidoff AM, Rice RP. Pneumatosis intestinalis. Surgical management and clinical outcome. Ann Surg. 1990 Aug;212(2):160-5.

13 Tak PP, Van Duinen CM, Bun P, Eulderink F, Kreuning J, Gooszen HG, et al. Pneumatosis cystoides intestinalis in intestinal pseudoobstruction. Resolution after therapy with metronidazole. Dig Dis Sci. 1992 Jun;37(6): 949-54.

14 Johnston BT, McFarland RJ. Elemental diet in the treatment of pneumatosis coli. Scand J Gastroenterol. 1995 Dec;30(12):1224-7.

15 Masterson JS, Fratkin LB, Osler TR, Trapp WG. Treatment of pneumatosis cystoides intestinalis with hyperbaric oxygen. Ann Surg. 1978 Mar;187(3):245-7.

16 Leffler DA, Lamont JT. Clostridium difficile infection. N Engl J Med. 2015 Apr 16;372(16):1539-48.

17 Lessa FC, Mu Y, Bamberg WM, Beldavs ZG, Dumyati GK, Dunn JR, et al. Burden of Clostridium difficile infection in the United States. N Engl J Med. 2015 Feb 26;372(9):825-34.

18 Chopra T, Chandrasekar P, Salimnia H, Heilbrun LK, Smith D, Alangaden GJ. Recent epidemiology of Clostridium difficile infection during hematopoietic stem cell transplantation. Clin Transplant. 2011 Feb;25(1): E82-7.

19 Cózar-Llistó A, Ramos-Martinez A, Cobo J. Clostridium difficile infection in special high-risk populations. Infect Dis Ther. 2016 Sep;5(3):253-69.

20 Louie TJ, Miller MA, Mullane KM, Weiss K, Lentnek A, Golan Y, et al. Fidaxomicin versus vancomycin for Clostridium difficile infection. N Engl J Med. 2011 Feb 3;364(5):422-31.

21 Montassier E, Gastinne T, Vangay P, Al-Ghalith GA, Bruley des Varannes S, Massart S, et al. Chemotherapydriven dysbiosis in the intestinal microbiome. Aliment Pharmacol Ther. 2015 Sep;42(5):515-28. 
22 Khan A, Raza S, Batul SA, Khan M, Aksoy T, Baig MA, et al. The evolution of Clostridium difficile infection in cancer patients: epidemiology, pathophysiology, and guidelines for prevention and management. Recent Pat Antiinfect Drug Discov. 2012 Aug;7(2):157-70.

23 Schmidt-Hieber M, Bierwirth J, Buchheidt D, Cornely OA, Hentrich M, Maschmeyer G, et al. Diagnosis and management of gastrointestinal complications in adult cancer patients: 2017 updated evidence-based guidelines of the Infectious Diseases Working Party (AGIHO) of the German Society of Hematology and Medical Oncology (DGHO). Ann Hematol. 2018 Jan;97(1):31-49.

24 Rodríguez Garzotto A, Mérida García A, Muñoz Unceta N, Galera Lopez MM, Orellana-Miguel MÁ, Díaz-García $\mathrm{CV}$, et al. Risk factors associated with Clostridium difficile infection in adult oncology patients. Support Care Cancer. 2015 Jun;23(6):1569-77.

25 Delgado A, Reveles IA, Cabello FT, Reveles KR. Poorer outcomes among cancer patients diagnosed with Clostridium difficile infections in United States community hospitals. BMC Infect Dis. 2017 23;17(1):448.

26 Cornely OA, Miller MA, Fantin B, Mullane K, Kean Y, Gorbach S. Resolution of Clostridium difficile-associated diarrhea in patients with cancer treated with fidaxomicin or vancomycin. J Clin Oncol. 2013 May 28;31(19): 2493-9.

27 Ms C, J K, Jo K, H P. Impact of malignancy on Clostridium difficile infection. Eur J Clin Microbiol Infect Dis. 2016 Jul 26;35(11):1771-6.

28 McDonald LC, Gerding DN, Johnson S, Bakken JS, Carroll KC, Coffin SE, et al. Clinical practice guidelines for Clostridium difficile infection in adults and children: 2017 update by the Infectious Diseases Society of America (IDSA) and Society for Healthcare Epidemiology of America (SHEA). Clin Infect Dis. 2018 Mar 19;66(7):e1-48.

29 Hochwald O, Shaoul R. Pneumatosis intestinalis caused by Clostridium difficile in a neutropenic child. Clin Gastroenterol Hepatol. 2007 Aug;5(8):A24.

30 Ha D, Tsai C-J. Pneumatosis intestinalis in a patient with recurrent Clostridium difficile infection [Internet]. 